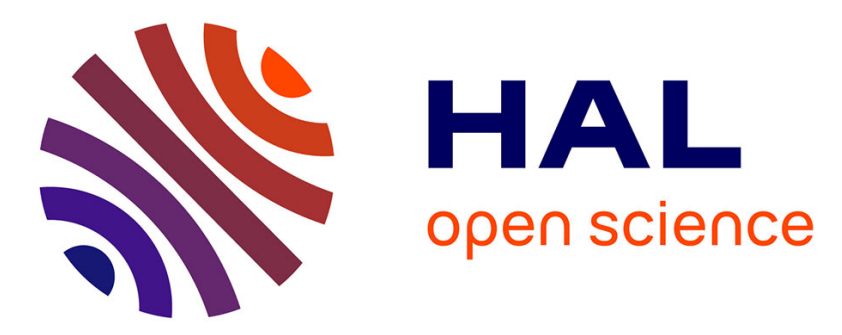

\title{
A fuzzy analytic hierarchy process for group decision making: application for embedding information on communicating materials
}

Sylvain Kubler, Alexandre Voisin, William Derigent, Eric Rondeau, André Thomas

\section{To cite this version:}

Sylvain Kubler, Alexandre Voisin, William Derigent, Eric Rondeau, André Thomas. A fuzzy analytic hierarchy process for group decision making: application for embedding information on communicating materials. IEEE International Conference on Communications, Computing and Control Applications, Dec 2012, Marseille, France. pp.270-276. hal-00762807

\section{HAL Id: hal-00762807 https://hal.science/hal-00762807}

Submitted on 8 Dec 2012

HAL is a multi-disciplinary open access archive for the deposit and dissemination of scientific research documents, whether they are published or not. The documents may come from teaching and research institutions in France or abroad, or from public or private research centers.
L'archive ouverte pluridisciplinaire HAL, est destinée au dépôt et à la diffusion de documents scientifiques de niveau recherche, publiés ou non, émanant des établissements d'enseignement et de recherche français ou étrangers, des laboratoires publics ou privés. 


\title{
A fuzzy analytic hierarchy process for group decision making: application for embedding information on communicating materials
}

\author{
Sylvain Kubler Alexandre Voisin William Derigent Éric Rondeau André Thomas \\ Centre de Recherche en Automatique de Nancy, Université de Lorraine, CNRS UMR 7039 \\ Campus Sciences, BP 70239, F-54506 Vandouvre-lès-Nancy Cedex, France, \\ FirstName.LastName@cran.uhp-nancy.fr
}

\begin{abstract}
It is not uncommon today to use intelligent products for ensuring an information continuum all along the product lifecycle. However, it is not that easy to identify what information should be stored on the product, in particular because of the large number of persons who are concerned by it. This paper proposes to use fuzzy AHP to select contextsensitive information from the database, that must be stored on the product. Through our approach, points of view from different actors are aggregated thanks to the fuzzy logic.
\end{abstract}

Keywords-Fuzzy AHP, group decision making, Intelligent product; Product Lifecycle Management; Data Dissemination

\section{INTRODUCTION}

In recent years, some authors have argued the use of intelligent products in the context of PLM (product lifecycle management) [1]. Indeed, a product moves through numerous companies through its lifecycle and technical, semantic and organizational interoperability between companies is not always ensured, thereby contributing to information loss. Considering the product as an information vector (in which data can be stored) should contribute to improved interoperability throughout the product lifecycle (PLC). However, most of the time, products only provide a network pointer to a linked database (e.g. a RFID tag) [1]. Moreover, this kind of product is still limited (e.g. risk of tag damage, small memory capacity,...).

As a result, we propose a new concept referred to as communicating material which considers the material as intrinsically communicating. Our recent works [2], [3], [4] published on this concept try to solve related research questions (product manufacturing, data processing,...). Among them, the automatic selection of relevant information from external databases to the material itself is an interesting topic. As a result, a data dissemination process is proposed to identify what the relevant information to users is and where it should be stored: on databases or on the products themselves? This process is applied each time a user wants to store information on the product and consists of two main steps :

1) selecting relevant information from the database that should be stored/replicated on the product [3],

2) storing information on the product and, subsequently, retrieving it. A communicating textile prototype is designed in [2] and a set of tools are developed in [4] to communicate with it.

${ }^{1} \mathrm{~A}$ huge quantity of RFID $\mu$ tags are spread in the textile.
This paper focuses on the first process step. The information relevance is dependent upon a variety of factors such as the user concerns, the product environment, etc. An approach is developed in [5] to select context-sensitive information by matching the context with data, which uses a Logical Data Model (LDM). Figure 1 gives insight into a part of a LDM, where one entity corresponds to a relational table as depicted with the entity/table Material. The attributes listed in each entity correspond to the table columns and each row is referred to as a tuple. For instance, Material has 3 attributes and 4 tuples. One data item corresponds to one table cell (cf. Figure 1).

The model developed in [5] assesses the relevance of all data items, from all tables. Their model uses the notion of priorities which are numerical values (either supplied or generated via observation and experimentation) assigned using a multifaceted evaluation of criteria. The higher the relevance value, the higher is the probability that this data item will be stored on the product. For instance, the data items $\mathrm{T}_{\mathrm{Mat}\{3,3\}}$ in Figure 1 is the most relevant compared to the others with a value of 0.40 .

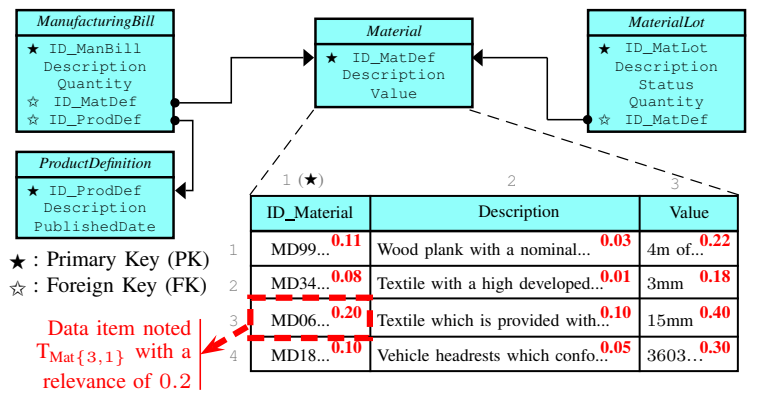

Figure 1. View of a Logical Data Model (LDM) and a relational table

The approach proposed in [5] has been applied in our previous work [3]. However, this approach does not offer possibilities to take into account several opinions and may turn out to be inappropriate to our context. Indeed, at a given moment of the PLC, several experts are concerned by selecting information that must be stored on the product, therefore generating different points of view. All points of view are legitimate and must be taken into account. Accordingly, this paper proposes an approach which combines the use of the analytic hierarchy process (AHP) with the fuzzy logic. AHP enables to handle the multi-criteria decision making (MCDM) problem and fuzzy logic enables to take into account multiple points of view. 
Section III briefly introduces fuzzy AHP. Section III introduces the fuzzy set semantic and representation used in our approach. Finally, section IV details the stages that compose our fuzzy AHP method (a case study is used as a "red line" to make the understanding easier).

\section{RELATED WORK}

AHP [6] deals with MCDM problems and is now used extensively in numerous sectors [7] by means of decision matrices expressing the expert points of view. However, classical AHP does not integrate an aggregation mechanism to allow the expression of multiple points of view. Therefore, to solve this issue, some works propose to use the fuzzy logic in conjunction with AHP.

Fuzzy set theory was introduced by [8] to deal with problems involving vagueness of human thinking. In a classical set $\mathcal{A}$, an element belongs entirely or not to $\mathcal{A}$. In a fuzzy set $\tilde{\mathcal{A}}$, an element can have different degrees of membership to $\tilde{\mathcal{A}}$.

The earliest work in fuzzy-AHP appeared in [9]. [10] introduced a new approach for handling fuzzy AHP, with the use of triangular fuzzy numbers for pairwise comparison scale and the use of the extent analysis method (FEAHP). In the literature, many works implement FEAHP. However, authors generally use the same membership functions (i.e. triangular) without thinking about the meaning of the information that must be modeled. Moreover, calculations in FEAHP are applied on particular points/parts of membership functions which can lead to information loss (i.e., loss of the form of the membership function).

In our study, we choose to use fuzzy AHP, on the one hand, in preserving the form of membership functions 2 and, on the other hand, by explaining clearly the semantic of each fuzzy set. To do so, specific tools/representations are used and are introduced in the next section.

\section{FUZZY SET SEMANTIC AND REPRESENTATION}

A solution that allows to preserve the form of membership functions consists in using the $\alpha$-cut representation. Section III-A introduces such a representation. The fuzzy set semantic used in our approach relies on two types of group consensus which are detailed in section III-B.

\section{A. $\alpha$-cut representation}

In a fuzzy set $\tilde{\mathcal{A}}$, an element can have different degrees of membership to $\tilde{\mathcal{A}}$. For each element of the referential $x$, a degree of membership to the fuzzy set is assigned, noted $\mu_{\tilde{\mathcal{A}}}(x)$. Thus, when $\mu_{\tilde{\mathcal{A}}}(x)=1, x$ belongs entirely to $\tilde{\mathcal{A}}$; when $\left.\left.\mu_{\tilde{\mathcal{A}}}(x) \in\right] 0 ; 1\right], x$ belongs more or less to $\tilde{\mathcal{A}}$. In our study, trapezoidal intervals are considered as depicted in Figure 2(a) noted $[a, b, c, d]$. Intervals $[b, c]$ and $[a, d]$ are respectively called the "kernel" and "support" of $\mu_{\tilde{\mathcal{A}}}(x)$.

As with crisp numbers, arithmetical operations can be defined to manipulate fuzzy sets. One technique consists in using a representation of fuzzy sets in the form of crisp intervals, called $\alpha$-cuts [8]. Then, operations are

\footnotetext{
${ }^{2}$ We think it is sensible to preserve the form of fuzzy sets when ranking alternatives.
}

applied on these intervals. An $\alpha$-cut level of $\tilde{\mathcal{A}}(\alpha \in] 0,1])$ corresponds to the crisp set $\mathcal{A}^{\alpha}$ such as :

$$
\begin{aligned}
\mathcal{A}^{\alpha} & \left.\left.=\left\{x \in \mathbb{R} \mid \mu_{\tilde{\mathcal{A}}}(x) \geq \alpha\right\} \quad \alpha \in\right] 0,1\right] \\
\text { by definition } \mathcal{A}^{0} & =\operatorname{support}(\tilde{\mathcal{A}})
\end{aligned}
$$

Figure 2(b) depicts the trapezoidal membership function $\mu_{\tilde{\mathcal{A}}}(x)$ by using $4 \alpha$-cut levels: $\alpha=\{0,0.33,0.66,1\}$. The use of crisp intervals makes it possible to preserve a calculation simplicity, but the membership function must be sampled as many times as $\alpha$-cut levels. In our study, we use $11 \alpha$-cut levels: $\alpha \in\{0,0.1,0.2, \ldots, 0.9,1\}$.

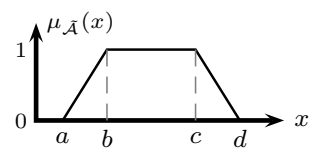

(a) Theoretical form

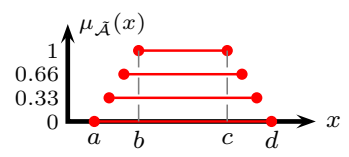

(b) $\alpha$-cut representation
Figure 2. Trapezoidal fuzzy sets: $[a, b, c, d]$

\section{B. Group consensus}

Two evaluation cases are identified in our study. Let us consider the evaluation of a student by his teachers:

- case $(a)$ : we seek to evaluate the students' skills in Mathematics by different teachers and exams. Thus we get several measures of a same object,

- case $(b)$ : we seek to evaluate the students' skills by teachers of Mathematics, English and Sport. The purpose here is to find a representation between several points of view on a same object.

The aggregation of different decision maker's point of view is thus performed according to the case, (a) or (b):

- consensus (a) : we propose to aggregate the crisp points of view and no information should be neglected. Accordingly, evaluation are aggregated via a fuzzy interval where all points of view form the kernel. However, we cannot rule out that the target value is located beyond the kernel (a new expert could give a new point of view outside the interval boundaries). We propose therefore to extend the support through a slope. Figure 3(a) illustrates the construction of the membership function on the basis of three distinct points of view noted $D_{1}, D_{2}$ and $D_{3}$,

- consensus (b) : as in consensus (a), no information should be neglected when aggregating points of view. The $x$-axis is a common semantic of points of view. Information complexity is also represented by an interval but, in opposition to consensus (a), the support is not extended because the addition of a new evaluation consists in adding a new point of view (e.g. add the Philosophy). Figure 3(b) illustrates the construction of the membership function on the basis of $D_{1}, D_{2}, D_{3}$.

\section{Process step 1 : Computation of DATA ITEM RELEVANCES USING FUZZY AHP}

In our study, fuzzy AHP consists of 5 stages (Figure 4):

1) breakdown the MCDM problem into a hierarchical structure, 


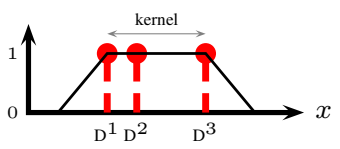

(a) consensus (a)

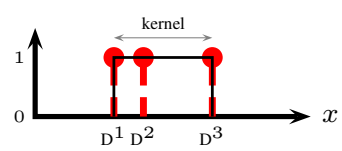

(b) consensus (b)
Figure 3. Aggregation of multiple points of view via 2 consensus

2) collection and aggregation of information for determining: i) fuzzy sets of alternatives with respect criteria, ii) the relative criteria importance,

3) creation of the fuzzy judgment matrix $\tilde{A}$ based on the fuzzy sets of alternatives with respect to criteria,

4) computation of the fuzzy performance matrix $\tilde{H}$ by synthesizing $\tilde{A}$ with the relative criteria importance,

5) alternative ranking. This step needs to aggregate the multi-criteria performance of alternatives in a fuzzy vector $\tilde{\mathcal{R}}$ and then, to rank alternatives.

(1)

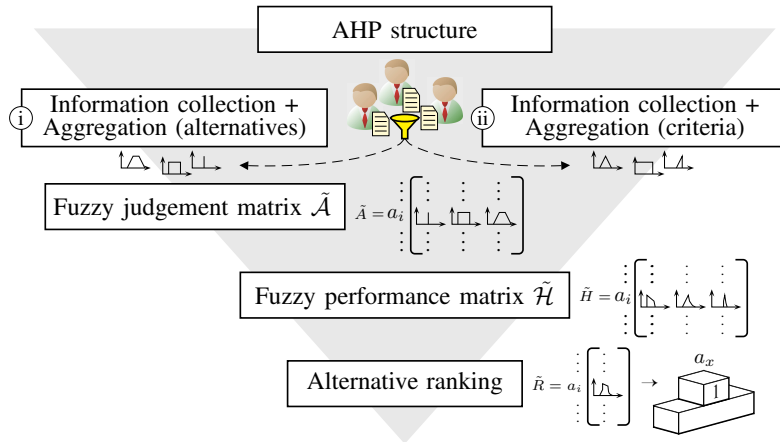

Figure 4. Fuzzy AHP consisting of 5 stages

To make the understanding easier, a scenario is considered in the rest of the paper whose parts are preceded by the symbol " $\rightleftharpoons$ ". This scenario relies on the piece of LDM introduced in Figure 1.

\section{A. Stage 1 : AHP structure}

Our MCDM problem is broken down into the hierarchical structure depicted in Figure 5, The alternatives are the data items ( $c f$. level 3) which must be assessed and ranked in term of relevancy ( $c f$. level 1). Three criteria are defined at level 2: Enumeration, Contextual and Data Size which are respectively abbreviated $\mathrm{C}_{e}, \mathrm{C}_{c}, \mathrm{C}_{s}$ and are detailed in the next stage.

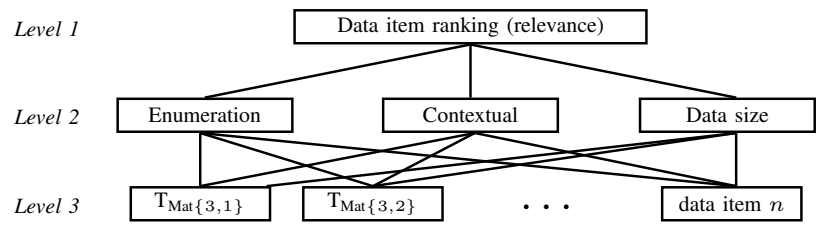

Figure 5. General architecture of the hierarchy

\section{B. Stage 2 : Information collection + Aggregation}

In this section, we provide information about the way to evaluate each alternative with respect to each criterion and the way to obtain the criteria importance.
1) Enumeration: Through this criterion, users may enumerate information they judge important to store on the product. To do so, each decision maker $p$ enumerates attributes from tables $(p \in\{1, \ldots, Q\}$ with $Q$ the total number of decision makers). Let $t$ a table from the LDM and $v$ an attribute of $t$. If $v$ is enumerated by $p$, the enumeration score $s^{p}(v, t)=0.5$ or 1 (depending on the preference intensity), otherwise 0 (see equation 1 ). Decision makers may come from different areas (production manager, shipping manager, etc.) and thereby, may want to store different information on the product. Accordingly, consensus (b) is used ( $c f$. Figure 3(a) and the set of points of view (noted $\mathcal{V}$ ) is aggregated through a classical interval in Equation 3. Finally, the score of a data item $l \in v$ with respect to $\mathrm{C}_{e}$, noted $\tilde{\Phi}_{e}(l)$, is equal to $\tilde{s}(v, t)$.

$$
\begin{aligned}
s^{p}(v, t) & =\left\{\begin{array}{ll}
1 & \text { enumerated (useful attribute) } \\
0.5 & \text { enumerated (interesting attribute) } \\
0 & \text { not enumerated (useless attribute) }
\end{array} \quad p=\{1, \ldots, Q\}\right. \\
\tilde{s}(v, t) & =[\min (\mathcal{V}) \max (\mathcal{V})], \quad \mathcal{V}=\left\{s^{1}(v, t), \ldots, s^{Q}(v, t)\right\}
\end{aligned}
$$

$\approx$ Three experts enumerate respectively attributes from Material (i.e. ID_Material, Description and value) as in Figure 6 ( $\mathrm{M}$ is the abbreviation of Material). Expert 1 judges "useful" information from attribute ID_Material: $s^{1}$ (ID_Material,M) $=1$ and "useless" information from attribute Description. Aggregation of the three expert's point of view, regarding each attribute, is performed based on Equation 3 Equation 4 details the calculation of $\tilde{s}$ (value, M) which is depicted in Figure 6 Fuzzy sets related to $\mathrm{T}_{\text {Mat }}\{3,1\}, \mathrm{T}_{\text {Mat }}\{3,2\}, \mathrm{T}_{\text {Mat }}\{3,3\}$ with respect to $\mathrm{C}_{e}$, noted $\tilde{\phi}_{e}\left(\mathrm{~T}_{\text {Mat }}\{3,1\}\right), \tilde{\phi}_{e}\left(\mathrm{~T}_{\text {Mat }}\{3,2\}\right), \tilde{\phi}_{e}\left(\mathrm{~T}_{\text {Mat }}\right.$ $\{3,3\})$, are respectively equal to $\tilde{s}($ ID_Material,M), $\tilde{s}($ Description, M) and $\tilde{s}$ (value, M) because they respectively belong to these three attributes.

$$
\tilde{s}(\text { value }, \mathrm{M})=[\min (1,1,0) \max (1,1,0)]=[0,1]
$$

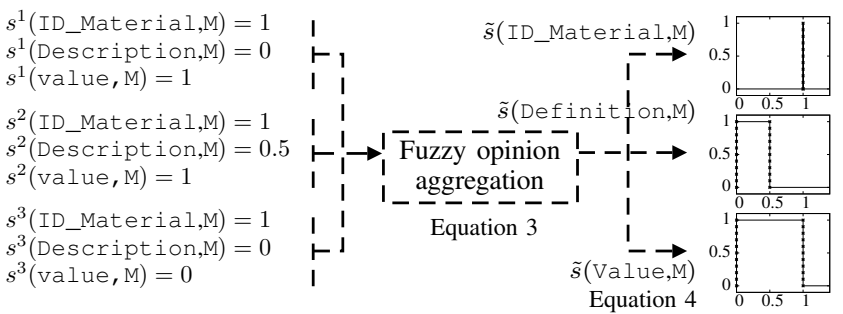

Figure 6. Fuzzy opinion aggregation related to Enumeration

2) Contextual: The enumerated adjustment gives users the freedom to specify information they want to store on the product. However, they could omit important information. Indeed, they might not be aware of all the data needed by the downstream actors (along the PLC). As a result, a new criterion referred to as contextual $\left(\mathrm{C}_{r}\right)$ is integrated in order to moderate and to balance $\mathrm{C}_{e}$. First, let us note that a multitude of information systems exist over the PLC (e.g. ERP, PDM, MES) which are not concerned by the same data (i.e. the same entities from the LDM). 


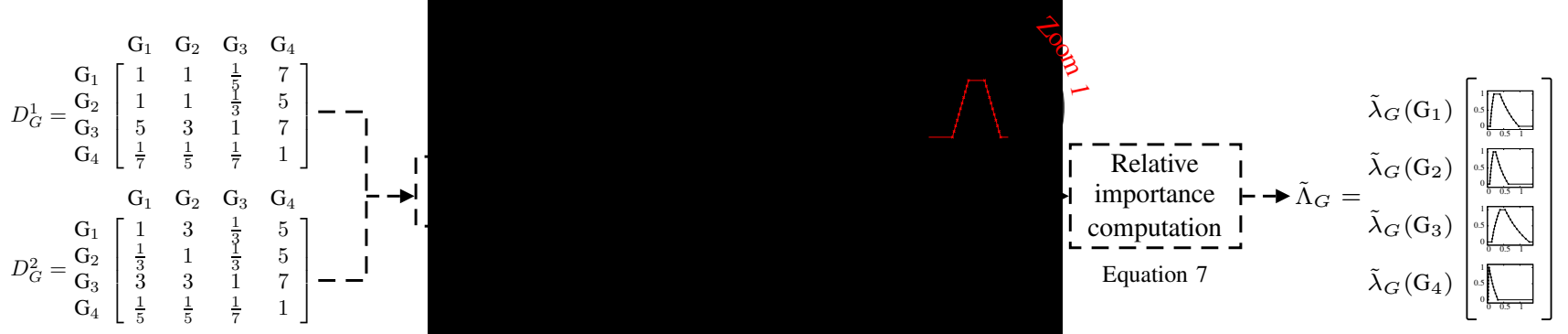

Figure 7. Pairwise comparisons between entity groups carried out by a group of experts, aggregation and computation of their relative importance

The idea is to identify specific "entity groups" through the LDM according to, for instance, the information systems and, therefore, to evaluate their importance over the PLC. In our study, each decision maker $p$ performs pairwise comparisons between entity groups as in Equation 5, with $Z$ the number of groups defined trough the LDM. The importance of entity group $i$ over entity group $j$ evaluated by the decision maker $p$ is noted $s_{i j}^{p}$. This evaluation is based on the 1 to 9 point scale from [6]: $\{1,3,5,7,9\}$. $s_{i j}^{p}=1$ means that groups $i$ and $j$ are equal in importance and $s_{p_{i j}}=9$ means that group $i$ is strongly favored $j$.

Experts who proceed to evaluations come from domains related to the information systems involved in PLC/PLM and have a same point of view on the criterion. As a result, it is necessary to aggregate the matrices $D_{G}^{p} \mid p=$ $\{1, \ldots, Q\}$ based on a consensus (b). All elements $s_{i j}^{p}$ are aggregated in a fuzzy set noted $\tilde{s}_{i j}$ through rules from Table [1]. We assume that experts agree on the sense of the importance $\left(\mathrm{G}_{i}>\mathrm{G}_{j}\right.$ or $\left.\mathrm{G}_{i}<\mathrm{G}_{j}\right)$. Matrix $\tilde{G}$ in Equation 6 synthesizes all fuzzy sets. Finally, the relative importance of each group $\mathrm{G}_{i}$ is computed in Equation 7 on the basis of $11 \alpha$-cuts (one $\alpha$-cut is noted $\lambda_{G}^{\alpha}\left(\mathrm{G}_{i}\right)$ ) and gives rise to a fuzzy set $\tilde{\lambda}_{G}\left(\mathrm{G}_{i}\right)$. These importances are synthesized in a fuzzy vector $\tilde{\Lambda}_{G}$ as in Equation 8 . If a data item $l$ is contained in a table included in $\mathrm{G}_{i}$, its score with respect to $\mathrm{C}_{c}$, noted $\tilde{\Phi}_{c}(l)$, is thus equal to $\tilde{\lambda}_{G}\left(\mathrm{G}_{i}\right)$.

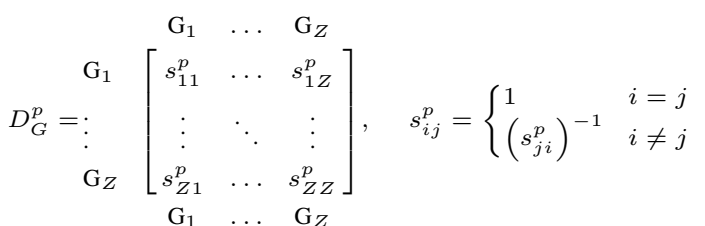

$$
\begin{aligned}
& \tilde{G}=\underset{\mathrm{G}_{Z}}{\mathrm{G}_{1}}\left[\begin{array}{ccc}
\tilde{s}_{11} & \ldots & \tilde{s}_{1 Z} \\
\vdots & \ddots & \vdots \\
\tilde{s}_{Z 1} & \ldots & \tilde{s}_{Z Z}
\end{array}\right] \\
& \lambda_{G}^{\alpha}\left(\mathrm{G}_{i}\right)=\frac{\sum_{j=1}^{Z} G^{\alpha}(i, j)}{\sum_{k=1}^{Z} \sum_{j=1}^{Z} G^{\alpha}(k, j)} \quad \forall \alpha=\{0,0.1,0.2, \ldots, 1\} \\
& \tilde{\Lambda}_{G}=\left[\tilde{\lambda}_{G}\left(\mathrm{G}_{1}\right), \tilde{\lambda}_{G}\left(\mathrm{G}_{2}\right), \ldots, \tilde{\lambda}_{G}\left(\mathrm{G}_{i}\right), \ldots, \tilde{\lambda}_{G}\left(\mathrm{G}_{Z}\right)\right]
\end{aligned}
$$

$\Rightarrow$ four entity groups are defined in the LDM. Two experts provide the pairwise comparisons in Figure 7 Expert 1 highly favors $\mathrm{G}_{1}$ over $\mathrm{G}_{4}\left(s_{14}^{1}=7\right)$ and expert 2 gives an importance slightly below $\left(s_{14}^{2}=5\right)$. Aggregation of $D_{G}^{1}$ and $D_{G}^{2}$ is then performed based on rules from

\footnotetext{
${ }^{3}$ we limit ourselves to extend the support to the graduation immediately superior or inferior of the Saaty scale because we consider five scale points and one graduation represents $25 \%$ of the scale.
}

Table I

RULE DEFINITION OF FUZZY NUMBERS FOR PAIRWISE COMPARISON

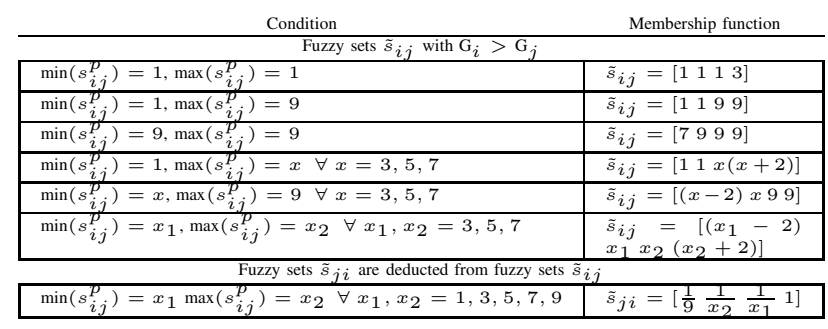

Table [1. Equation 9 details the aggregation computation related to elements $s_{14}^{1}$ and $s_{14}^{2}$ whose the resulting fuzzy set $\tilde{s}_{14}$ is depicted in Figure 7 (zoom 1). Finally, the relative importance of each group $\mathrm{G}_{i}$ is computed via Equation 7 and are synthesized in $\tilde{\Lambda}_{G}$. We can see that information from $\mathrm{G}_{3}$ is the most important at this stage of the PLC4 follows up by $G_{1}, G_{2}$ and $G_{4}$. If we assume that Material $\in \mathrm{G}_{1}$, therefore, fuzzy sets of $\mathrm{T}_{\text {Mat }}\{3,1\}, \mathrm{T}_{\text {Mat }}\{3,2\}, \mathrm{T}_{\text {Mat }}\{3,3\}$ with respect to $\mathrm{C}_{c}$, noted $\tilde{\phi}_{c}\left(\mathrm{~T}_{\text {Mat }}\{3,1\}\right), \tilde{\phi}_{c}\left(\mathrm{~T}_{\text {Mat }}\{3,2\}\right), \tilde{\phi}_{c}\left(\mathrm{~T}_{\text {Mat }}\{3,3\}\right)$ are equal to $\tilde{\lambda}_{G}\left(\mathrm{G}_{1}\right)$.

$s_{14}^{1}=7, s_{14}^{2}=5 \underset{\text { Table }]}{\left[\left(x_{1}-2\right) x_{1} x_{2}\left(x_{2}+2\right)\right]} \tilde{s}_{14}=[(5-2) 57(7+2)]$ (9)

3) Data size: This criterion aims to favor the storage of information on the product according to the data size. Since products are often memory-constrained, data relevance should decrease when data size increases. Such a behavior can be obtained via Equation 10, with $d$ the size of a data item $l$ and $k^{p}$ a constant adjusted by the expert $p$. Figure 8 shows two functions considering two different $k^{p}$. It can be observed that the smaller $k^{p}$ is, the bigger the data authorized to be stored on the product is (data with sizes $>60$ bytes are neglected when $k=1.08$ ).

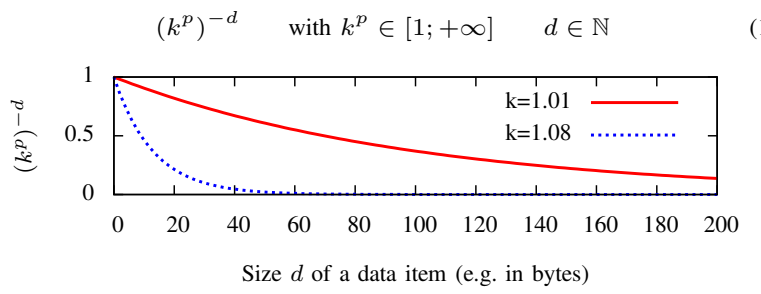

Figure 8. Adjustment of $k^{p}$ performed by a decision maker $p$

\footnotetext{
${ }^{4}$ The more the surface of the membership function form, the more the fuzzy set is important in our case.
} 


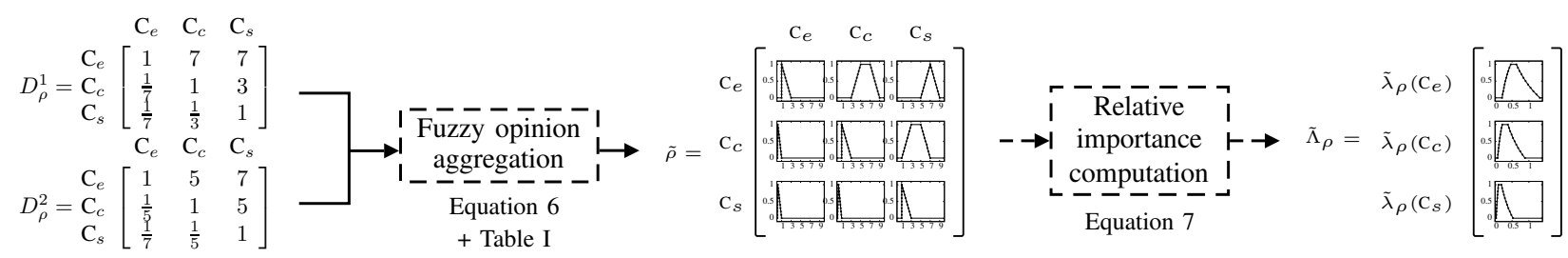

Figure 10. Pairwise comparisons between criteria carried out by a group of experts, aggregation and computation of their relative importance

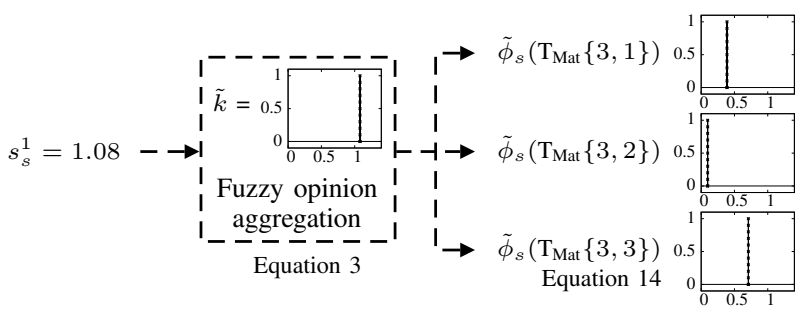

Figure 9. Fuzzy opinion aggregation related to Data Size

As previously, experts may have difference points of view on this criterion and may adjust differently $k^{p}$. Let us consider an expert 1 who is interesting to a high quantity of tuples (e.g., expert 1 enumerates many attributes) and an expert 2 who is interesting to a small quantity. As a result, expert 1 may intend to store on the product small data items to get a "complete/diversified" view of the quantity, while expert 2 wants to authorize the storage of big data items (i.e. $k^{1}>k^{2}$ ). Accordingly, the consensus (a) is used for aggregating the different $k$ values in a fuzzy set $\tilde{k}$ as in Equation 11. Finally, the fuzzy set of a data item $l$ with respect to $\mathrm{C}_{s}$, noted $\tilde{\Phi}_{s}(l)$, is computed in Equation 12 .

$$
\begin{aligned}
\tilde{k} & =[\min (\mathcal{V}) \max (\mathcal{V})], \quad \mathcal{V}=\left\{k^{1}, \ldots, k^{Q}\right\} \\
\tilde{\phi}_{s}(l) & =\tilde{k}^{-d}=\left[\begin{array}{ll}
\max (\mathcal{V})^{-d} & \min (\mathcal{V})^{-d}
\end{array}\right]
\end{aligned}
$$

$\Leftrightarrow$ a unique expert 1 adjusts the coefficient $k_{p}$ at 1.08 to neglect data items $>60$ bytes. The fuzzy set resulting of the aggregation is equal to a singleton set of 1.08 as detailed in Equation 13 and illustrated in Figure 9 The fuzzy set of $\mathrm{T}_{\text {Mat }}\{3,1\}$ with respect to $\mathrm{C}_{s}$, noted $\tilde{\Phi}_{s}\left(\mathrm{~T}_{\text {Mat }}\{3,3\}\right)$ is computed in Equation 14 and illustrated in Figure 9 This figure also provides the fuzzy sets $\tilde{\Phi}_{s}\left(\mathrm{~T}_{\text {Mat }}\{3,1\}\right)$ and $\tilde{\Phi}_{s}\left(\mathrm{~T}_{\text {Mat }}\{3,2\}\right)$ which depends on both data item sizes.

$$
\begin{aligned}
\tilde{k}=[\min (1.08) \max (1.08) & =\left[\begin{array}{ll}
1.08 & 1.08
\end{array}\right] \\
\tilde{\phi}_{s}\left(\mathrm{~T}_{\mathrm{Mat}}\{3,1\}\right)=\left[\begin{array}{ll}
1.08^{-4} & 1.08^{-4}
\end{array}\right] & =\left[\begin{array}{ll}
0.73 & 0.73
\end{array}\right]
\end{aligned}
$$

4) Criteria importance: Experts specify the criteria importance via pairwise comparisons as in $\mathrm{C}_{r}$. The relative importance of each criterion, noted $\tilde{\lambda}_{\rho}\left(\mathbf{C}_{x}\right)$, is computed based on the same rules (Table $\Pi$ ) and equations (6 to 8 ).

$\triangle 2$ experts perform pairwise comparisons in Figure 7 and the relative importance of criteria are synthesized by $\Lambda_{\rho}$. We can see that $\mathrm{C}_{e}$ is the most important criterion (i.e. experts take the liberty of choosing information to store on the product at the expense of the others criteria).

\footnotetext{
${ }^{5} \mathrm{~T}_{\text {Mat }}\{3,3\}$ is a character string " $15 \mathrm{~mm}$ " and 1 ASCII character is coded on 1 byte. That is why the power is equal to " 4 " in Equation 14
}

\section{Stage 3 : Fuzzy judgment matrix $\tilde{A}$}

After getting the fuzzy set of each alternative $l$ $(l \in\{1, \ldots, n\})$ with respect to each criterion $x(x \in$ $\left.\left\{\mathrm{C}_{e}, \mathrm{C}_{c}, \mathrm{C}_{s}\right\}\right)$, noted $\tilde{\phi}_{x}(l)$, the fuzzy judgment matrix $\tilde{\mathcal{A}}$ is created as depicted in Figure 11

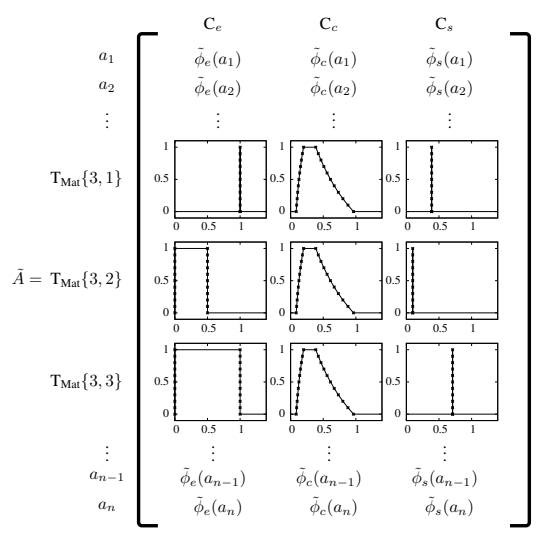

Figure 11. Fuzzy judgment matrix $\tilde{A}$

\section{Stage 4 : Fuzzy performance matrix $\tilde{H}$}

At this stage, only scores of alternatives with respect to criteria are taken into account, without considering the relative importance of criteria. As a result, we synthesize the fuzzy matrix $\tilde{A}$ with the criteria importance in a fuzzy performance matrix $H$. The performance score $\tilde{h}_{x}(l)$ consists in multiplying the fuzzy set $\tilde{\phi}_{x}(l)$ by the criterion importance itself $\lambda_{\rho}\left(\mathbf{C}_{x}\right)$. This multiplication is carried out based on their $\alpha$-cut levels as detailed in Equation 15 . Finally, the matrix $\tilde{H}$ is obtained as depicted in Figure 12 , in which we highlight the multiplication $\tilde{h}_{e}\left(\mathrm{~T}_{\text {Mat }}\{3,1\}\right)$.

$$
\tilde{h}_{x}(l)=\phi_{x}^{\alpha}(l) \cdot \lambda_{\rho}^{\alpha}\left(\mathrm{C}_{x}\right) \quad \forall \alpha=\{0,0.1, \ldots, 1\}
$$

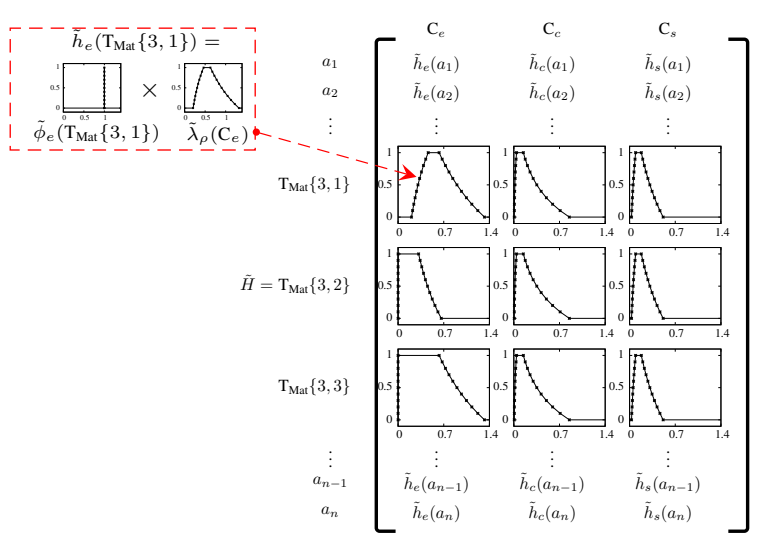

Figure 12. Fuzzy performance matrix $\tilde{H}$ 


\section{E. Stage 5 : Alternative ranking}

This stage ranks alternatives according to their fuzzy sets but, until now, 1 alternative has 3 fuzzy sets ( $c f$. Figure 12). As a result, it is necessary to proceed to the multicriteria aggregation and then, to compare alternatives.

In our approach, the multi-criteria aggregation consists, for each alternative, in summing the 3 fuzzy sets based on their $\alpha$-cuts as detailed in Equation 16. Figure 13 illustrates the summing operation $\tilde{r}\left(\mathrm{~T}_{\text {Mat }}\{3,1\}\right)$.

$$
r^{\alpha}(l)=\sum_{x \in\{e, c, m, s\}} h_{x}^{\alpha}(l) \quad \forall \alpha=\{0,0.1, \ldots, 1\}
$$

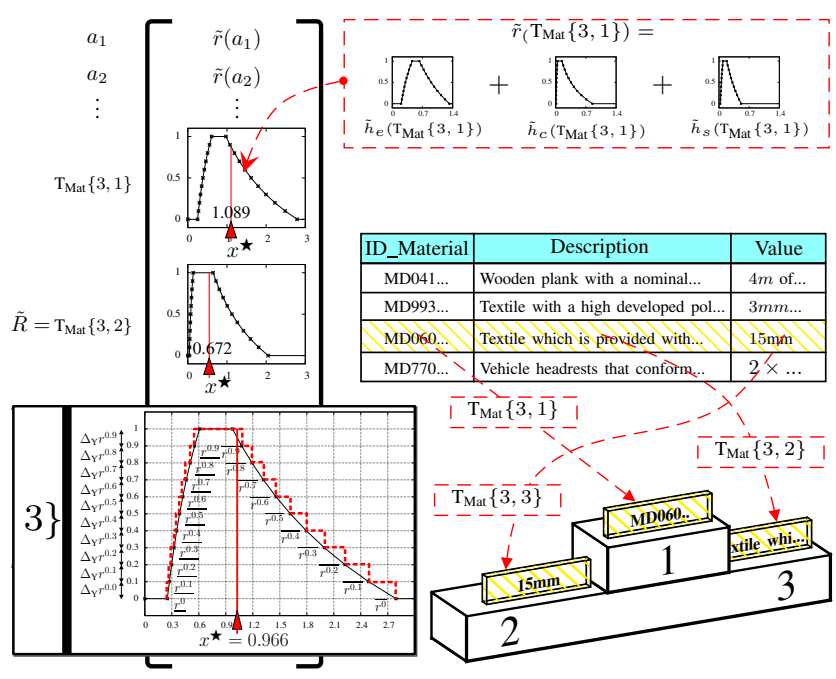

Figure 13. Alternative ranking according to the $\mathrm{CoG}$

In our study, the approach based on the center of gravity $(\mathrm{CoG})$, originally proposed by Yager [11], is used for ranking alternatives according to their $\alpha$-cut levels. This technique computes, for each fuzzy number, an index noted $x^{\star}$ which is used to compare and to rank alternatives. This index is a crisp value located on the $x$ axis and is computed via Equation 17 However, since our approach uses $\alpha$-cut representations, the integral functions are therefore discretized and are an approximation of the result. We propose to compute the superior approximation result as in Equation 17, where $m$ is the number of $\alpha$-cuts (i.e., $\left.\alpha=\{\underbrace{0}_{\alpha_{0}}, \ldots, \underbrace{0.8}_{\alpha_{\mathrm{m}-2}}, \underbrace{0.9}_{\alpha_{\mathrm{m}-1}}\}\right), \overline{r^{\alpha}}$ and $\underline{r^{\alpha}}$ respectively indicate the minimal and the maximal values of the $\alpha$-cut and $\Delta_{\mathrm{Y}} r^{\alpha}$ corresponds to the level difference between the $\alpha$-cut and $\alpha+1$-cut levels. All these notations are detailed in Figure 13 with regard to $\tilde{r}\left(\mathrm{~T}_{\text {Mat }}\{3,3\}\right)$.

$$
x^{\star}=\frac{\int \mu_{\tilde{\mathcal{A}}}(x) \times x d x}{\int \mu_{\tilde{\mathcal{A}}}(x) d x} \approx \frac{\sum_{\alpha=\left\{\alpha_{0} \ldots \alpha_{\mathrm{m}-1}\right\}}\left[\frac{r^{\alpha}+\overline{r^{\alpha}}}{2} \cdot \Delta_{\left.\mathrm{Y}^{r^{\alpha}}\right]}\right.}{\sum_{\alpha=\left\{\alpha_{0} \ldots \alpha_{\mathrm{m}-1}\right\}} \Delta_{\mathrm{Y}} r^{\alpha}}
$$

It can be observed that the CoG of $\mathrm{T}_{\text {Mat }}\{3,1\}\left(x^{\star}=\right.$ 1.089) is higher than the CoG of $\mathrm{T}_{\text {Mat }}\{3,2\}\left(x^{\star}=0.672\right)$ and $\mathrm{T}_{\text {Mat }}\{3,3\}\left(x^{\star}=0.966\right)$. As a result, $\mathrm{T}_{\text {Mat }}\{3,1\}$ gets a better ranking ( $c f$. podium in Figure 13) and is stored in priority on the product.
Finally, the list of data items is ranked and is stored on the communicating material according to its available memory space (thanks to the process step 2). In our application, around 500 data items are ranked (representing $\approx 30$ Mbytes). Our textile can only embed 4 Mbytes and only the first 70 data items can therefore be stored on it.

\section{CONCLUSION}

For years, the use of intelligent products have been used to create an information continuum all along the product life cycle. However, it is not that easy to identify what information should be stored on the product. As a result, we propose a data dissemination process to select contextsensitive information from databases to the products. The information relevance depends on many factors leading to a multi-criteria decision making (MCDM) problem. To handle the MCDM problem, we propose an approach based on fuzzy AHP which is particularly interesting compared to conventional approaches (e.g., FEAHP) because, the membership function forms are preserved through the use of $\alpha$-cut representations and, the fuzzy set semantic is analyzed in view of our application (two consensus are proposed to aggregate multiple points of view).

\section{REFERENCES}

[1] C. Y. Wong, D. Mcfarlane, A. A. Zaharudin, and V. Agarwal, "The Intelligent Product Driven Supply Chain," in International Conference on Systems, Man and Cybernetics, 2002, pp. 4-6.

[2] S. Kubler, W. Derigent, A. Thomas, and É. Rondeau, "Prototyping of a communicating textile," in International Conference on Industrial Engineering and Systems Management, 2011, pp. 1333-1342.

[3] — , "Information dissemination process for contextaware products," in 14th Symposium of Information Control Problems in Manufacturing, 2012.

[4] - "Storage of information on manufactured products using "communicating materials"," in AI Problems and Approaches for Intelligent Environments (AI@IE), 2012.

[5] D. Chan and J. Roddick, "Context-sensitive mobile database summarisation," in 26th Australasian computer science conference, vol. 16, 2003, pp. 139-149.

[6] T. Saaty, The Analytic Hierarchy Process. New York: McGraw-Hill, 1980.

[7] O. Vaidya and S. Kumar, "Analytic hierarchy process: An overview of applications," European Journal of operational research, vol. 169, no. 1, pp. 1-29, 2006.

[8] L. Zadeh, "Fuzzy sets," Information and Control, vol. 8, pp. 338-353, 1965.

[9] P. van Laarhoven and W. Pedrycz, "A fuzzy extension of saaty's priority theory," Fuzzy Sets and Systems, vol. 11, no. 1, pp. 199-227, 1983.

[10] D.-Y. Chang, "Applications of the extent analysis method on fuzzy ahp," European Journal of Operational Research, vol. 95, no. 3, pp. 649-655, 1996.

[11] R. Yager, "On a general class of fuzzy connectives," Fuzzy sets and Systems, vol. 4, no. 3, pp. 235-242, 1980. 